\title{
In situ electron-beam lithography of deterministic single-quantum-dot mesa-structures using low-temperature cathodoluminescence spectroscopy
}

Cite as: Appl. Phys. Lett. 102, 251113 (2013); https://doi.org/10.1063/1.4812343

Submitted: 15 April 2013 • Accepted: 11 June 2013 • Published Online: 26 June 2013

M. Gschrey, F. Gericke, A. Schüßler, et al.
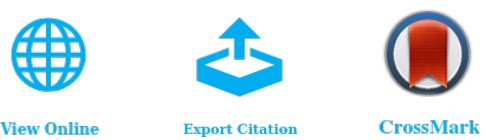

\section{ARTICLES YOU MAY BE INTERESTED IN}

Resolution and alignment accuracy of low-temperature in situ electron beam lithography for nanophotonic device fabrication

Journal of Vacuum Science \& Technology B 33, 021603 (2015); https://doi.org/10.1116/1.4914914

On-demand generation of background-free single photons from a solid-state source

Applied Physics Letters 112, 093106 (2018); https://doi.org/10.1063/1.5020038

Advanced in-situ electron-beam lithography for deterministic nanophotonic device processing

Review of Scientific Instruments 86, 073903 (2015); https://doi.org/10.1063/1.4926995

母QBLOX

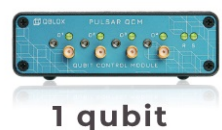

Shorten Setup Time Auto-Calibration More Qubits

Fully-integrated Quantum Control Stacks Ultrastable DC to $18.5 \mathrm{GHz}$ Synchronized $<<1$ ns Ultralow noise

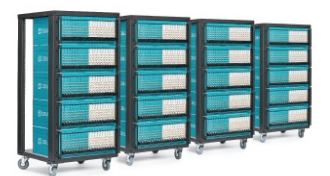

100s qubits

visit our website > 


\title{
In situ electron-beam lithography of deterministic single-quantum-dot mesa-structures using low-temperature cathodoluminescence spectroscopy
}

\author{
M. Gschrey, F. Gericke, A. Schüßler, R. Schmidt, J.-H. Schulze, T. Heindel, S. Rodt, \\ A. Strittmatter, and S. Reitzenstein ${ }^{\text {a) }}$ \\ Institut für Festkörperphysik, Technische Universität Berlin, Hardenbergstraße 36, D-10623 Berlin, Germany
}

(Received 15 April 2013; accepted 11 June 2013; published online 26 June 2013)

\begin{abstract}
We report on the deterministic fabrication of sub- $\mu \mathrm{m}$ mesa-structures containing single quantum dots (QDs) by in situ electron-beam lithography. The fabrication method is based on a two-step lithography process: After detecting the position and spectral features of single InGaAs QDs by cathodoluminescence (CL) spectroscopy, circular sub- $\mu$ m mesa-structures are defined by highresolution electron-beam lithography and subsequent etching. Micro-photoluminescence spectroscopy demonstrates the high optical quality of the single-QD mesa-structures with emission linewidths below $15 \mu \mathrm{eV}$ and $\mathrm{g}^{(2)}(0)=0.04$. Our lithography method has an alignment precision better than $100 \mathrm{~nm}$ which paves the way for a fully deterministic device technology using in situ CL lithography. (C) 2013 AIP Publishing LLC. [http://dx.doi.org/10.1063/1.4812343]
\end{abstract}

The development of optical quantum devices based on single-photon emitters such as semiconductor quantum dots (QDs) embedded into microcavities has revolutionized the field of quantum optics in solid-state physics. ${ }^{1,2}$ Initially, and to a large extent even today, such devices rely on the selection of statistically grown QDs in a random process. As a result, the yield of functional devices is very low, typically below $1 \%$, which hinders further development toward a practical technology platform. Moreover, the statistical spatial and spectral coupling of self-assembled QDs to the optical modes in microcavity systems impedes systematic studies of lightmatter interaction effects. For these reasons, huge efforts have been directed toward a deterministic device technology. One route to achieve this goal is based on the site-controlled growth of QDs and their integration into nanophotonic devices, and enormous progress has been achieved in this field in recent years. ${ }^{3-9}$ However, site-controlled QDs still suffer from a degraded optical quality in terms of emission linewidths and quantum efficiency as compared to standard QDs based on the Stranski-Krastanow growth mode. ${ }^{10}$ In addition, their emission energy, and thus the spectral matching in QD-microcavities, is hardly controlled during growth. In an alternative and fully deterministic approach, in situ optical lithography has been established to select the position and emission energy of target QDs before defining spatially and spectrally matched microcavity structures. ${ }^{11}$ This very attractive technology platform has the drawback that optical lithography has limited lateral resolution of a few hundred $\mathrm{nm}$ at best.

In contrast, electron-beam lithography (EBL) can achieve a resolution of a few $\mathrm{nm}$ which is a prerequisite for the realization of advanced nanodevices. A combination of EBL and cathodoluminescence (CL) is very promising for site-selective exposure of the resist with respect to the position of optically active nanostructures like, e.g., QDs. In the following, this technique will be dubbed cathodoluminescence lithography (CLL). Such an approach has been used to deterministically contact single nanowires in Ref. 12, but

${ }^{\text {a)} E l e c t r o n i c ~ a d d r e s s: ~ s t e p h a n . r e i t z e n s t e i n @ p h y s i k . t u-b e r l i n . d e ~}$ without the need for a spectral selection of the wires. Extending CLL with respect to nanophotonic devices could strongly boost the development of QD micro- and nanocavities by enabling the fabrication of fully deterministic structures with $\mathrm{nm}$ accuracy and a very flexibel device design in combination with spectral matching.

In this letter, we report on a deterministic nanophotonic device technology based on low-temperature CLL. Our technology platform combines the advantages of CL spectroscopy and high-resolution electron-beam writing into a unique lithography method for nanophotonic devices. To demonstrate the power of this method, we take advantage of the high spatial resolution of CL to precisely determine the location of target QDs and to fabricate nm-scaled mesa structures spatially aligned to the selected QDs. Optical studies on the patterned samples prove the high yield and accuracy of this lithography technique and show its suitability for the fabrication of high-quality nanophotonic structures. Our CLL method has high potential to pave the way for a new generation of quantum devices where nanoscale cavity structures can be aligned to quantum emitters with very high precision in order to realize, e.g., non-classical light sources or a single-QD laser for on-chip optical networks. Moreover, it could also be used for the deterministic fabrication of nanoelectronics devices such as QD-based flash-memories relying on a precise positioning of single QDs in the active layer.

As an in situ lithography method, CLL is performed without the need of alignment markers or a transfer of the sample into another system for electron-beam writing. This leads to a potentially more reliable and simpler process flow as compared to other EBL approaches using for instance optical spectroscopy to identify the position of QDs relative to alignment markers, which allows one to retrieve their positions in a subsequent EBL step after transferring the sample. ${ }^{13}$ The process flow of our approach is depicted in Figure 1. After spin coating the QD-sample with standard electronbeam resist (polymethylmethacrylat, 950 K PMMA) we perform CL spectroscopy to build a luminescence map from individual QDs to measure their emission spectra and to 


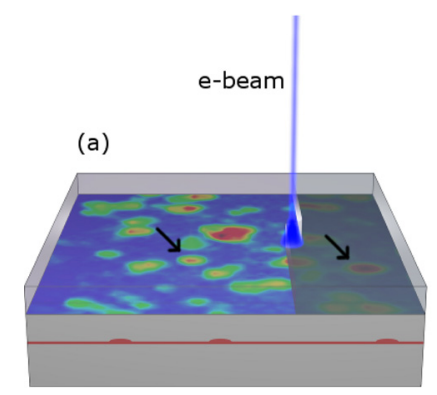

(c)
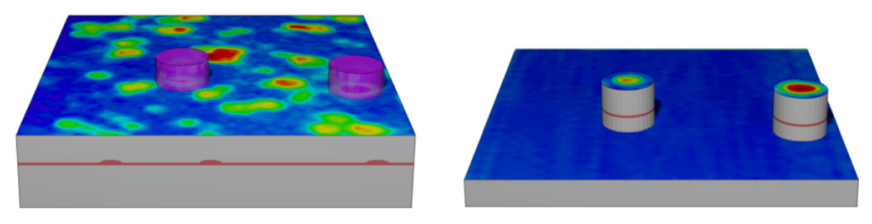

FIG. 1. Schematic view of the CLL process flow. After selecting target QDs by CL-mapping (a), sub- $\mu$ m mesa structures are written into the PMMA resist (b) which acts as a negative resist by inverting at the large doses used in the lithography step. After developing the resist (c) the electron-beam defined structures are transferred into the semiconductor material by dry chemical etching which results in single-QD mesa structures (d).

determine their spatial positions with an accuracy of less than $50 \mathrm{~nm}$ (Fig. 1(a)). In this step, it is crucial to ensure an homogenous exposure without inverting the resist. In accordance with Ref. 12, the resist was found to sustain low temperatures very well and to be less sensitive for electron doses as compared to room temperature. Once suitable QDs have been identified in the resulting CL map (indicated by black arrows), circular patterns that are spatially aligned to the selected QDs are written into the resist (Fig. 1(b)), where the mesa-diameter is controlled precisely by the applied dose. After developing the resist (Fig. 1(c)) the remaining PMMA acts as etching mask in a subsequent plasma etching step (Fig. $1(\mathrm{~d})$ ) which results in single-QD nanostructures as will be shown below.

Most critical in CLL is the careful choice of the CLscanning parameters in the QD-selection process and of the exposure parameters in the subsequent lithography step. In fact, during CL-scanning it is necessary to achieve a good balance between a low exposure dose (as a function of beam current, acceleration voltage, and integration time per spectrum) and sufficiently high CL signal. It turned out that PMMA, which is a positive resist, cannot be used in the normal mode because the lowest possible dose $\left(<500 \mu \mathrm{C} / \mathrm{cm}^{2}\right)$ during CL scanning already fully cracks the polymer and would lead to a complete removal of the exposed resist in the development step. This is illustrated in Fig. 2 where the remaining thickness after cold developing (IPA, $-35^{\circ} \mathrm{C}$ ) an exposed layer of $200 \mathrm{~nm}$ PMMA on a GaAs sample is shown after scanning with an acceleration voltage of $10 \mathrm{kV}$ and increasing exposure times. The range of resulting exposure doses $\left(0.5-7 \mathrm{mC} / \mathrm{cm}^{2}\right)$ in which the CL-mapping is performed is indicated in green. For larger doses the resist becomes inverted and acts as a negative resist, so that the remaining thickness increases up to about $150 \mathrm{~nm}$ for a dose of $40 \mathrm{mC} / \mathrm{cm}^{2}$. This is exploited in the lithography step which is performed for doses in the range of $14-45 \mathrm{mC} / \mathrm{cm}^{2}$ as indicated in blue.
Our CLL method is demonstrated by fabricating circular sub- $\mu \mathrm{m}$ mesa structures aligned to pre-selected InGaAs QDs. The sample was grown by metal-organic chemical vapor depositon in an AIXTRON 200/4 machine on a $3^{\prime \prime}$ GaAs (001) wafer using standard precursors. First, $500 \mathrm{~nm}$ of GaAs was deposited followed by $30 \mathrm{~nm}$ of $\mathrm{AlGaAs}$ as diffusion barrier for charge carriers and $150 \mathrm{~nm}$ of GaAs. The selforganized QDs were formed at a temperature of $500^{\circ} \mathrm{C}$ during a growth interruption of $35 \mathrm{~s}$. Finally the QDs were capped by $150 \mathrm{~nm}$ of GaAs, $27 \mathrm{~nm}$ of AlGaAs as second diffusion barrier, and $5 \mathrm{~nm} \mathrm{GaAs}$ as top layer. Due to intrinsic carbon doping during growth, a p-type background doping of $\approx 1 \times 10^{17} \mathrm{~cm}^{-3}$ is present in the sample. For AFM measurements, a second layer of QDs was added on top of the sample with the same growth conditions as the first layer. At helium temperatures, the QDs emit at a wavelength of around $950 \mathrm{~nm}$. The QD density was estimated to be about $1 \times 10^{9} \mathrm{~cm}^{-2}$. After the sample growth we used spin coating to deposit a homogenous, $200 \mathrm{~nm}$ thick layer of PMMA on the sample. Then a $5 \mathrm{~mm} \times 5 \mathrm{~mm}$ sample was transferred into the CL-system in order to perform CL-mapping for the selection of suitable QDs. A typical CL-map of a $22 \mu \mathrm{m} \times 30 \mu \mathrm{m}$ sample area is shown in Fig. 3(a), clearly marking the positions of single QDs by bright spots. In this example, we selected three free-standing QDs labeled 1 to 3 for in situ lithography. The corresponding electron-beam writing was performed directly after the CL-mapping without moving the sample. The position of each of the QDs was precisely determined by applying a 2D-fitting routine to the intensity distribution in the CL-map for the spectral window depicted by the blue, dashed line in Fig. 3(d). At each position, a circular pattern of nominal $500 \mathrm{~nm}$ in diameter was written. Afterwards, the resist was cold developed and the inverted resist above the chosen QDs was used as an etch mask in the subsequent etching step using an ICP-RIE plasma $\left(\mathrm{BCl}_{2}+\mathrm{Cl}_{2}+\mathrm{Ar}\right)$ etcher. Figure 3(c) shows a high-resolution SEM image of the fully processed mesas. In order to demonstrate the deterministic integration of single QDs into sub- $\mu \mathrm{m}$ mesa structures, we performed another CL-mapping on the processed sample. The corresponding CL-intensity map is depicted in Fig. 3(b)

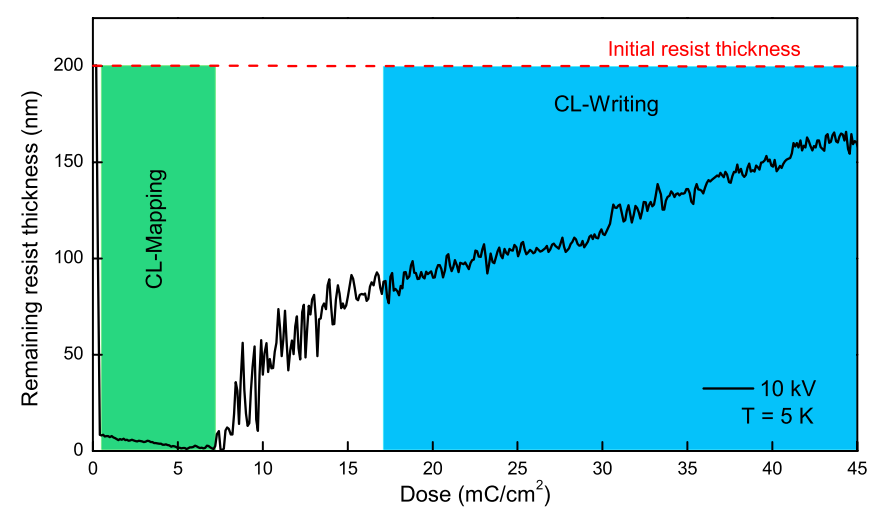

FIG. 2. Characteristics of the e-beam resist (PMMA) in the CLL-process at low temperature $(5 \mathrm{~K})$ and an acceleration voltage of $10 \mathrm{kV}$. The remaining thickness of the resist after development is depicted as a function of the exposure dose. The resist with an initial thickness of $200 \mathrm{~nm}$ acts as positive resist up to about $7 \mathrm{mC} / \mathrm{cm}^{2}$ and has negative characteristics for larger doses by inverting. This feature is exploited in CLL where CL-mapping is performed at intermediate doses as indicated in green and electron-beam writing is done at larger doses of up to $45 \mathrm{mC} / \mathrm{cm}^{2}$ as indicated in blue. 

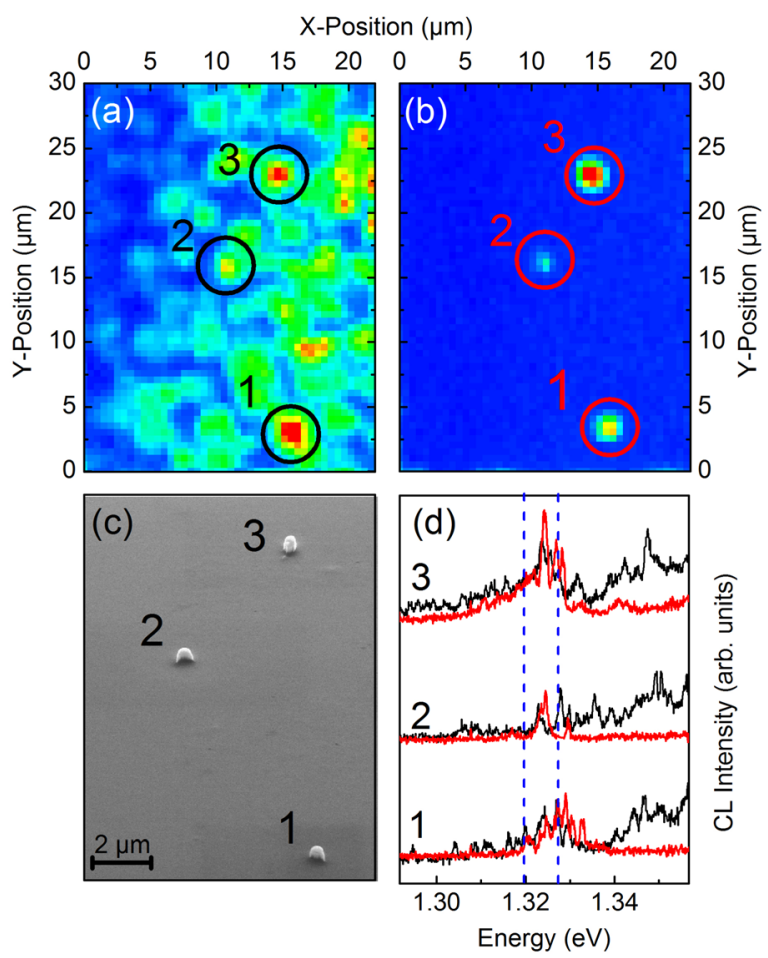

FIG. 3. Low-temperature CL-intensity maps (linear scale) obtained (a) during CL spectroscopy and (b) after the processing of single-QD mesa structures for an acceleration voltage of $10 \mathrm{kV}$ and a beam current of $0.5 \mathrm{nA}$. The QDs selected during the CL-mapping (labelled 1-3) are integrated deterministically into structures with diameters below $500 \mathrm{~nm}$. (c) High-resolution SEM image recorded under an angle of $60^{\circ}$ of the processed sample. (d) CLemission spectra of the selected QDs recorded during CL-mapping (black) and on the final mesa structures (red). The blue, dashed lines indicate the spectral window, which was used for the 2D-fitting routine to determine the QD position and for which the integrated intensity is displayed in (a) and (b).

and demonstrates that all three mesa structures with preselected QDs are optically active. Associated CL-spectra recorded during the mapping step (black curves) and after etching (red curves) are shown in Fig. 3(d). The single-QD linewidths of about $0.9 \mathrm{meV}$ are limited by the spectral resolution of the CL setup. The fact that the spectral features are reproduced by the processed sample clearly demonstrates the deterministic integration of selected QDs into the sub- $\mu \mathrm{m}$ mesa structures.

The reliability of our CLL method was checked by fabricating several circular mesa structures with diameters of $800 \mathrm{~nm}, 450 \mathrm{~nm}$, and $350 \mathrm{~nm}$. For the largest diameter $89 \%$, i.e., 38 out of 43 mesas, of the pre-selected QDs showed unaltered luminescence as compared to measurements before fabrication of the mesas. The $450 \mathrm{~nm}$-sized structures had such a yield of $67 \%$ and a yield of $50 \%$ was achieved for the $350 \mathrm{~nm}$-sized mesas. Here, we have to take into account that QDs implemented in small mesa structures are influenced by fluctuating charge distributions at defects in the dot's environment or at the lateral sidewalls, which results in spectral diffusion ${ }^{14}$ and increased emission linewidths. ${ }^{15}$ Hence, further optimization of the etch process might increase our yield. The positioning accuracy was checked by reference samples as follows: A metallic shadow mask, fabricated with the aid of dispersed $100 \mathrm{~nm}$ polystyrene nano-spheres, was used as a spotty luminescence grid placed on top of a quantum-well sample. By
CLL cross-markers were fabricated around the luminescence spots. The accuracy of aligning the cross-markers relative to the mask openings was better than $100 \mathrm{~nm}$ as confirmed by SEM measurements.

To further study the optical quality of the single-QD mesas, we performed high-resolution micro-photoluminescence $(\mu \mathrm{PL})$ spectroscopy at low temperature $(\mathrm{T}=10 \mathrm{~K})$, during which the sample was optically excited by a $\mathrm{HeNe}$ laser. PL was collected by a $20 \times$ microscope objective with a numerical aperture of 0.4 , dispersed by a monochromator and detected by a Si-charge-coupled-device camera. Figure 4 displays a $\mu \mathrm{PL}$-spectrum of the CLL-processed mesa containing QD \#3 (cf. Fig. 3) for an excitation power of $1.65 \mu \mathrm{W}$. By analyzing the spectral diffusion of the emission lines, we could verify that all lines belong to one and the same QD. ${ }^{16}$ We observe narrow emission lines resulting from the recombination of the exciton $(\mathrm{X})$, biexciton $(\mathrm{XX})$, and singly-charged exciton $\left(\mathrm{X}^{+}\right)$states of the single QD. Thereby, the transitions were identified by power- as well as polarization-dependent measurements. The remaining emission lines are most probably due to transitions of charged excitonic complexes as a consequence of the background doping in the sample. By fitting a Voigt profile to the emission lines of $\mathrm{X}, \mathrm{XX}$ and $\mathrm{X}^{+}$, at which the Gaussian part was set to the spectral resolution of our setup $(23 \mu \mathrm{eV})$, we extract Lorentzian linewidths of $\gamma_{X}=15 \mu \mathrm{eV}, \gamma_{X X}=13 \mu \mathrm{eV}$ and $\gamma_{X+}=9 \mu \mathrm{eV}$ underlining the high optical quality of the single-QD mesa structure. Furthermore, photon autocorrelation measurements were carried out on the $\mathrm{X}^{+}$-emission for an excitation power of $75 \mathrm{nW}$. The emission line was spectrally selected by a monochromator and coupled into a fiber-based Hanbury-Brown and Twiss setup (HBT). It consists of a multimode fiber-beamsplitter $(50 \mu \mathrm{m}$ corediameter, split ratio 50:50) together with two $\tau$-SPAD-50 (PicoQuant) avalanche photo diodes featuring a timing resolution down to $350 \mathrm{ps}$. In order to shift the detectors

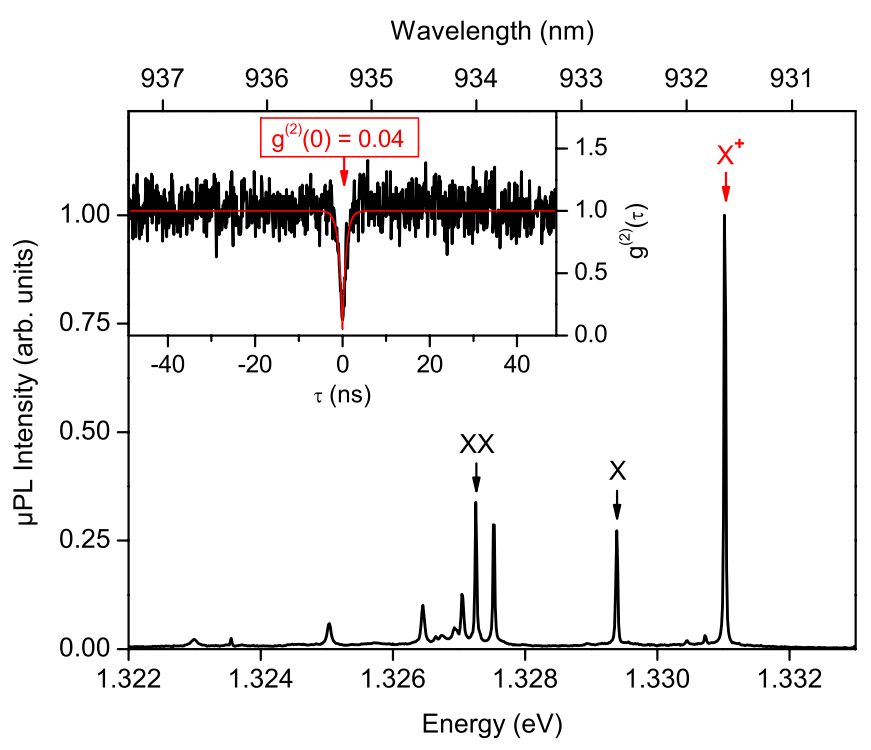

FIG. 4. $\mu \mathrm{PL}$ emission spectrum of QD \#3 $(\mathrm{T}=10 \mathrm{~K})$. Exciton $(\mathrm{X})$, biexciton $(\mathrm{XX})$, and singly charged exciton $\left(\mathrm{X}^{+}\right)$emission lines are identified and the linewidths down to $9 \mu \mathrm{eV}$ clearly reflect the high optical quality of the single-QD mesa structures. Inset: Photon auto-correlation measurement carried out on the $\mathrm{X}^{+}$-emission revealing a $g^{(2)}(0)$-value as low as 0.04 . 
cross-talk away from the zero-delay point, a $25 \mathrm{~m}$ multimode fiber-delay is attached symmetrically to both arms of the HBT. Time correlated single-photon counting is performed by a PicoHarp 300 module with a time bin width of 4 ps. The resulting histogram of the photon auto-correlation function $\mathrm{g}^{(2)}(\tau)$ is shown in the inset of Figure 4, where a time binwidth of $128 \mathrm{ps}$ was chosen. A fit to the raw data yields a value of $g^{(2)}(0)=0.04$, proving the quantum nature of emission with a strong suppression of two-photon emission events.

In conclusion, we have demonstrated a lithography technique which allows for the deterministic selection and processing of sample-areas containing single QDs with high yield and high optical quality. Our lithography approach is based on combined high-resolution CL-spectroscopy and in situ electron-beam writing of nanophotonic structures. The high quality and enormous potential of the approach was demonstrated by fabricating deterministic single-QD mesa structures with sub- $\mu$ m dimensions. It combines the pre-selection of quantum emitters by means of their lateral and spectral positions with the ability of patterning lowdimensional structures with nm precision and thus will pave the way for a new generation of fully deterministic quantum devices with unprecedented complexity and quality.

We acknowledge support from Deutsche Forschungsgemeinschaft (DFG) through SFB 787 "Semiconductor Nanophotonics: Materials, Models, Devices."
${ }^{1}$ A. J. Shields, Nat. Photonics 1, 215 (2007).

${ }^{2}$ S. Reitzenstein, IEEE J. Sel. Top. Quantum Electron. 18, 1733 (2012).

${ }^{3}$ O. G. Schmidt, Lateral Alignment of Epitaxial Quantum Dots (Springer, Berlin, 2007).

${ }^{4}$ C. Schneider, M. Strauß, T. Sünner, A. Huggenberger, D. Wiener, S. Reitzenstein, M. Kamp, S. Höfling, and A. Forchel, Appl. Phys. Lett. 92, 183101 (2008).

${ }^{5}$ C. Schneider, A. Huggenberger, T. Sünner, T. Heindel, M. Strauß, S. Göpfert, P. Weinmann, S. Reitzenstein, L. Worschech, M. Kamp et al., Nanotechnology 20, 434012 (2009).

${ }^{6}$ J. Skiba-Szymanska, A. Jamil, I. Farrer, M. B. Ward, C. A. Nicoll, D. J. P. Ellis, J. P. Griffiths, D. Anderson, G. A. C. Jones, D. A. Ritchie et al., Nanotechnology 22, 065302 (2011).

${ }^{7}$ K. D. Jöns, P. Atkinson, M. Müller, M. Heldmaier, S. M. Ulrich, O. G. Schmidt, and P. Michler, Nano Lett. 13(1), 126 (2012).

${ }^{8}$ A. Strittmatter, A. Schliwa, J.-H. Schulze, T. D. Germann, A. Dreismann, O. Hitzemann, E. Stock, I. A. Ostapenko, S. Rodt, W. Unrau et al., Appl. Phys. Lett. 100, 093111 (2012).

${ }^{9}$ W. Unrau, D. Quandt, J.-H. Schulze, T. Heindel, T. D. Germann, O. Hitzemann, A. Strittmatter, S. Reitzenstein, U. Pohl, and D. Bimberg, Appl. Phys. Lett. 101, 211119 (2012).

${ }^{10}$ F. Albert, S. Stobbe, C. Schneider, T. Heindel, S. Reitzenstein, S. Höfling, P. Lodahl, L. Worschech, and A. Forchel, Appl. Phys. Lett. 96, 151102 (2010).

${ }^{11}$ A. Dousse, L. Lanco, J. Suffczyński, E. Semenova, A. Miard, A. Lemaître, I. Sagnes, C. Roblin, J. Bloch, and P. Senellart, Phys. Rev. Lett. 101, 267404 (2008).

${ }^{12}$ F. Donatini and L. S. Dang, Nanotechnology 21, 375303 (2010).

${ }^{13}$ T. Kojima, K. Kojima, T. Asano, and S. Noda, Appl. Phys. Lett. 102, 011110 (2013).

${ }^{14}$ V. Türck, S. Rodt, R. Heitz, O. Stier, M. Straßburg, U. W. Pohl, and D. Bimberg, Physica E 13, 269 (2002).

${ }^{15}$ M. Bayer and A. Forchel, Phys. Rev. B 65, 041308 (2002).

${ }^{16}$ S. Rodt, A. Schliwa, K. Pötschke, F. Guffarth, and D. Bimberg, Phys. Rev. B 71, 155325 (2005). 